\title{
Modelling of high temperature storage systems for latent heat
}

\author{
Andreas Stückle \\ Institute of Technical Thermodynamics, German Aerospace Center (DLR) \\ Pfaffenwaldring 38-40, 70569 Stuttgart, Germany \\ andreas.stueckle@dlr.de
}

\begin{abstract}
There is a huge demand for heat storages for evaporation applications. Thermal storage systems are used to increase the efficiency of thermal systems by an improved adaption of energy availability and energy demand.

In this paper a possible solution for modular storage systems from $200-600{ }^{\circ} \mathrm{C}$ and pressures up to 100 bar is presented. The application of steam as a working medium requires the availability of isothermal storage if charging/discharging should take place at almost constant pressure. The saturation temperature range is between $200^{\circ} \mathrm{C}$ and $320^{\circ} \mathrm{C}$. Therefore nitrate salts are used as phase change material (PCM). The solution developed at DLR is characterized by a modular concept of tube register storages surrounded by both sensible and latent heat storage material.
\end{abstract}

The focus in this paper is on modelling of the PCM storage. A model is introduced for melting and freezing of the PCM. To perform with an acceptable heat transfer rate inside the PCM, fins are used to increase the overall thermal conductivity. Instead introducing mean storage material parameters, like thermal conductivity or specific heat capacity, the geometry of the finned tube is modelled by using discrete elements. Therefore the model allows detailed studies on heat transfer during space and time. The fin design can be varied in order to find an optimal configuration. A set of partial differential equations is created and solved. When considering a stand alone system, that means tube, fin and PCM, without a connection to other components, investigation is quite effective. In case of the PCM storage there is the big advantage, compared with a sensible regenerator, that the almost constant fluid temperature, when evaporating or condensing, leads to a uniform temperature distribution in fluid flow direction. Therefore only a very rough discretisation in axial direction is needed, what even allows bonding with other components e.g. from the Modelica Fluid Library.

Sensible storages as they are used for preheating and superheating have a characteristic temperature gradient in axial direction. To describe their thermal behaviour concentrated models, using dimensionless numbers, are used.

Keywords: latent heat; storage system; high temperature

\section{Introduction}

The range for heat storage for evaporation applications is wide. One of the big topics today is the equipment of thermal solar power plants for direct steam generation [1] with thermal storage to increase their total electricity output. Direct steam generation means that the heat transfer fluid is water and steam. Heat accumulators are a key for electricity suppliers to guarantee safety of supply and to achieve good results.

The DLR is working on solutions for storage systems from $200-600^{\circ} \mathrm{C}$ and pressures up to 100 bar. The saturation temperature range of the saturated steam is between $200^{\circ} \mathrm{C}$ and $320^{\circ} \mathrm{C}$. Due to the saturation temperature, the melting range of the $\mathrm{PCM}$ is between $200^{\circ} \mathrm{C}$ and $320^{\circ} \mathrm{C}$. Therefore, nitrate salts are used.

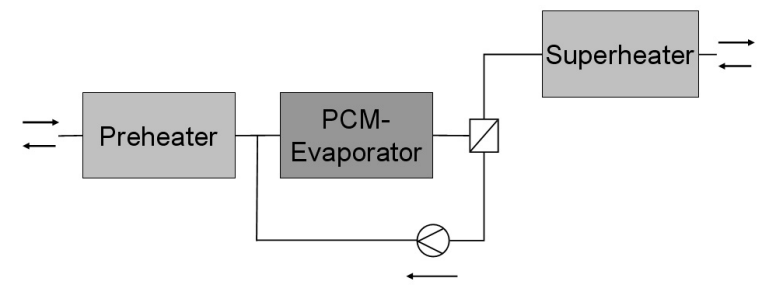

Figure 1: Modular Storage System 
The storage systems developed at DLR are characterized by a modular configuration of sensible and latent heat accumulators (see Figure 1). The sensible accumulators are made of a special high temperature concrete. Both species are based on a tube register surrounded by the storage material. The tube register separates the heat transfer fluid from the storage material. According to the Rankine process, a storage system has to deliver superheated steam when discharging. Therefore, the storage system for direct steam generation is divided into modules. Feed water is heated up close to the evaporation point in a preheater storage. Evaporation takes place in the evaporator system consisting of the evaporator PCMstorage, a steam separator and a recirculation pump [2]. The last part of the process is the superheating of the saturated steam. Preheater and superheater storage are both sensible accumulators.

When charging the system, the fluid flows in the opposite direction. The superheated steam is cooled down in the superheater storage; thereafter condensed in the evaporator storage; finally the liquid water is cooled down in the preheater.

Heat transfer in solids is characterized by partial differential equations. Additionally, a model to describe the melting and solidifying of the PCM was developed. Convectional heat transfer as well as condensation and evaporation processes affect the heat transfer at the tube's inner surfaces and are described by a Nusselt correlation for evaporation or condensation [3]. Also, pressure losses are calculated by correlations. A steam- and water model is used to compute state variables. Conservation laws for heat and mass flow have to be solved.

A storage system for direct steam generation is characterized by a modular design. That means that there will not be a monolithic storage block but several units. Therefore, an object oriented approach is convenient, particularly if the storage model will be connected in the future with a power block or a collector field model. Furthermore, peripheral equipment such as pumps and valves have to be modelled and controlled. Because of its interdisciplinary features and modular character, Modelica is used.

The aim of this work is to provide a tool to design and optimize storage systems and analyze especially transient effects.

\section{PCM Storage Model}

\subsection{Discrete Storage Models}

In the first package of this work, discrete models of "Storage-Tubes" were developed. Connecting these elements in series and in parallel forms a whole storage module [4]. The storage models are twodimensional formations fragmented into differential elements. That means that the cylindrical storage element is differentiated in axial and radial directions. The third dimension, which is the angle dependence around the tube axis, is neglected because it is assumed that the heat transfer is axially symmetric. This assumption is particularly proper for vertical tube registers. Vertical design is applied for high temperature PCM storages because of various constructive reasons.

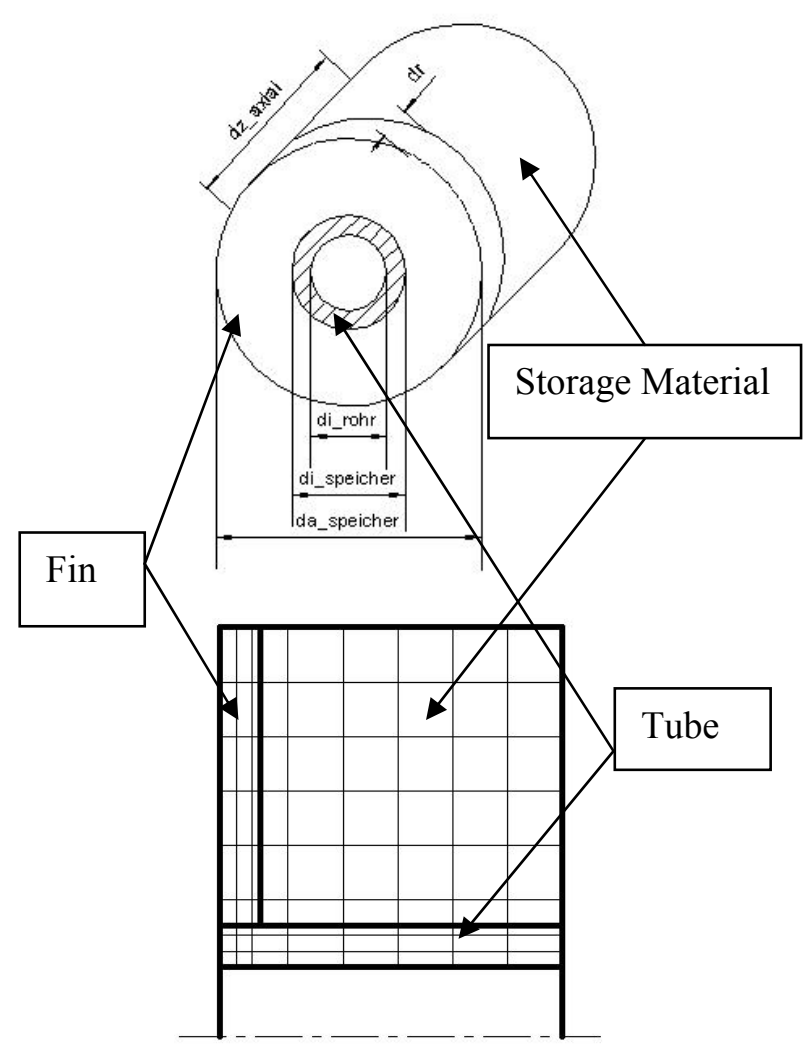

Figure 2: Model and two-dimensional grid of a storage tube

The discrete elements as depicted in Figure 2 are all composed in the same structure. Each element is defined by coordinates $\mathrm{x} 1$ and $\mathrm{x} 2$. In the Modelica language, indices are used to call the element.

Mass, heat capacity, substance values and state variables are concentrated in a centre point of the finite volume element, which is from here on called cell. 
This concentrated mass is surrounded by thermal resistivities. They depend on the heat conductance value of the cell, the distance between the centre point and the heat transfer across the cross-sectional area between two cells (see Figure 3).

The cells are coupled by "connectors" to transfer the flow variable "heat flow rate" and the state variable "temperature".

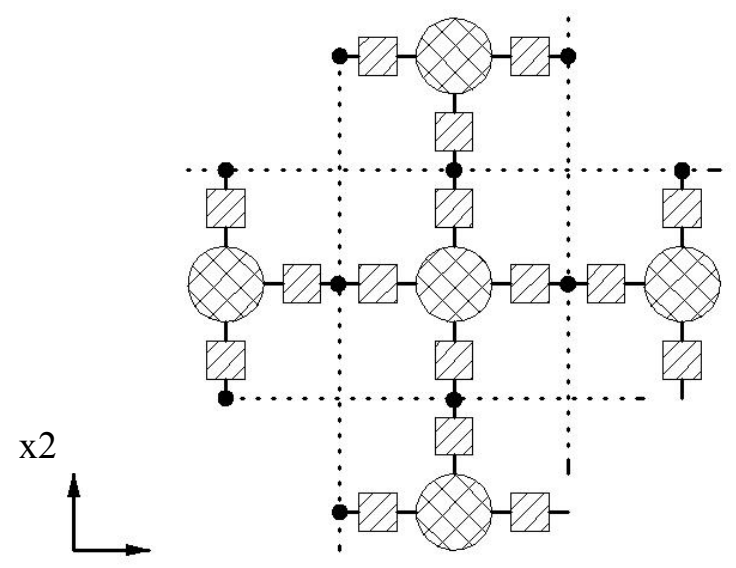

$\mathrm{x} 1$

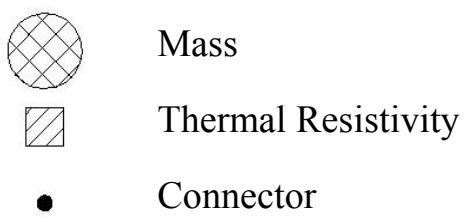

Figure 3: Structure of Discrete Elements

\subsection{Energy Balance}

The following differential equation describes the enthalpy change of a cell (left side) by the heat flow over the cell's borders (right side).

$$
\frac{d}{d t} \int_{V} \rho \cdot c_{p} \cdot T \cdot d V=-\int_{S} \vec{J} \cdot \hat{n} \cdot d S
$$

The heat transfer mechanism on the right side can be convection, conduction or even radiation.

In this paper, only convection on the inside of the storage tubes and conduction inside the material is considered. This leads to the following form whereas for the conduction the heat flow is the product of the cross section, the heat transfer coefficient and the temperature gradient perpendicular to the cross section. For the cylindrical shape, a form factor is used.

At the inner tube side the convective heat coefficient is derived from a Nusselt-correlation.

$$
-\left.\int_{S} \vec{J} \cdot \hat{n} \cdot d S \Rightarrow A \cdot k \frac{\partial T}{\partial x}\right|_{S}
$$

\subsection{PCM Model}

To model melting and freezing of cells, a model with a specific heat capacity for the solid phase and liquid phase is used. In theory, the enthalpy of a pure medium rises in a step change when melting or freezing. From experiments, we know that in engineering applications with nitrate salts there will not be a phase change at a certain temperature but over a small temperature interval of ca. $1 \mathrm{~K}$.

This effect is also useful for numerical calculations where step changes should be avoided to guarantee convergence and to increase calculation performance. Good performance is even reached with a range of $0.1 \mathrm{~K}$. As there is no sense in engineering applications to declare such a small range, the melting range is assumed to be $1 \mathrm{~K}$.

An effective specific heat capacity for the melting range is defined by the specific enthalpy of fusion divided by the temperature melting range.

$$
c_{p, \text { melt }}=\frac{h_{\text {melt }}}{\Delta T_{\text {melt }}}
$$

The following figure shows how the enthalpy of a PCM increases with the rise of temperature.

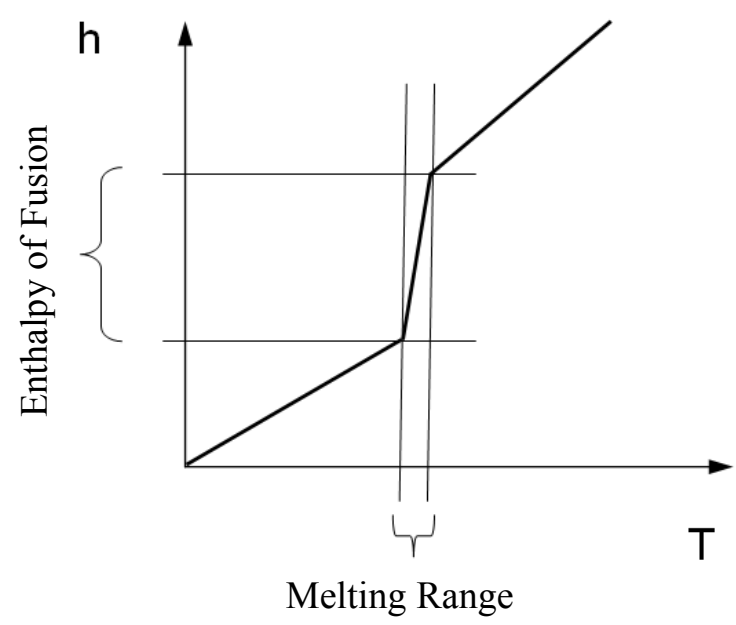

Figure 4: Enthalpy of the PCM 
The PCM is treated as a solid even for the liquid phase. That means that there are no convective heat transfer mechanisms if the PCM is liquid. They would improve the performance when charging by mass transfer inside the liquid phase.

The output performance of a thermal storage is critical because the discharge rating is usually higher or equal to the charging rating. When discharging the PCM storage, the PCM first solidifies at the surface of the tube and the fins. Therefore, the heat transfer mechanism is conduction.

\subsection{Comparison with a Fluent Model}

Plausibility of the model was checked by a 3-D Fluent calculation (see Figure 5, the broken line is the Fluent result, the continuous line from Modelica). The Fluent standard PCM Model is used. As in Modelica convectional heat transfer in the liquid phase is neglected.

The temperatures of the cells lying on the line from the chamfer between tube and fin to the cell with the longest distance from tube and fin were compared (see Figure 2). The rugged temperatures from the Modelica model result from the coarse discretisation, the Modelica grid consists of only 90 knots, but the total time to melt the PCM completely is almost the same. For this case, the PCM was molten in Fluent within $8514 \mathrm{~s}$ compared to $8726 \mathrm{~s}$ in Fluent, which is less than $2.5 \%$ difference.

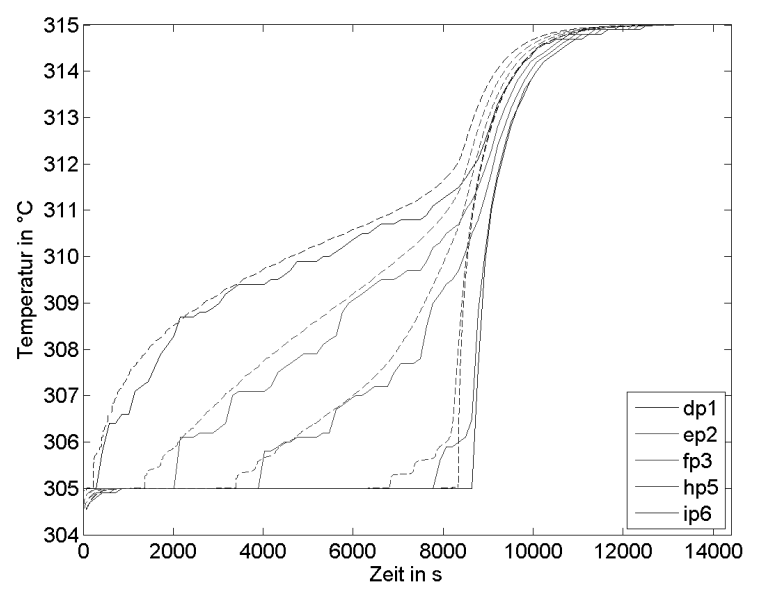

Figure 5: Comparison of the Modelica Model with Fluent

The Fluent calculation took several hours whereas the Modelica model only takes a few seconds. The advantage of the Modelica model is the much higher performance, which even allows coupling of the model into a system simulation.

\section{Application}

\subsection{Dimensioning of Fins and Tubes}

These models are used to configure fins for different applications, especially finned tubes and fins for a claimed power in-/output by best utilization.

For evaporating water, high heat flux rates are needed. Therefore, different kinds of fins can be used to conduct the heat flow from the solidifying PCM into the storage material or backwards.

Performance of fins of different materials and geometries is shown. The melting and freezing of the PCM is examined, especially the melting front, which controls power in- and output. The melting front can be visualized as a two dimensional plot using Matlab as depicted in Figure 6.

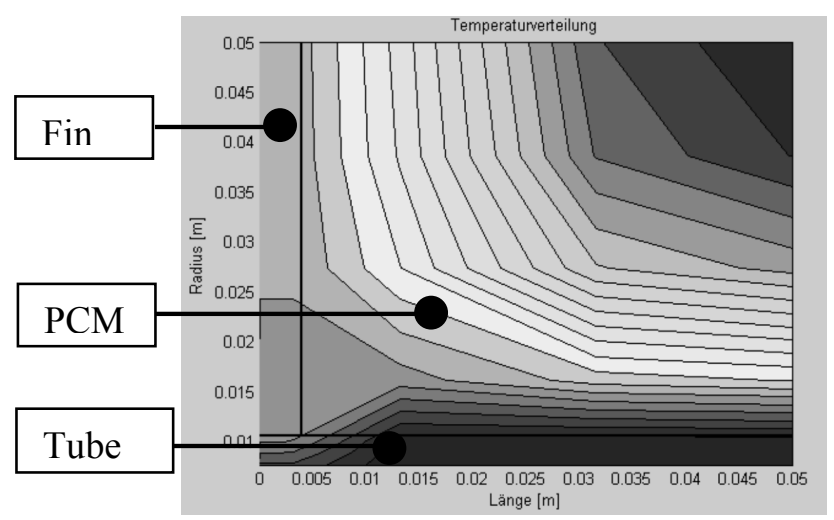

Figure 6: Temperature Field displayed in Matlab

In the following figure, two fins are compared. Integral heat flow rates are depicted. A cycle of complete melting and solidification is shown. The storage was initialized with a homogenous temperature $1 \mathrm{~K}$ below the melting temperature. Therefore, before melting $0.5 \mathrm{~K}$ of sensible heat is needed. Heating was stopped until the temperature of all cells was at least $0.5 \mathrm{~K}$ above the melting range. That means all cells are liquid and slightly superheated. Cooling is done with the same gradient and until the PCM is subcooled $0.5 \mathrm{~K}$. As expected, the model shows a symmetric behaviour.

The peak in heat flow rates at the beginning of charging and discharging are due to the highest temperature gradients at these moments.

$\dot{Q}_{\text {total }}$, the total transferred heat flow rate, is the heat flow rate transferred between the heat transfer fluid and the inner surface of the tube. $\dot{Q}_{\text {Fin }}$ is the flow rate between the fin and PCM and analogously $\dot{Q}_{\text {Tube }}$ between the tube and PCM. 
The importance of the heat conducting role of the fins in PCM evaporator applications is underlined when considering that about $85 \%$ of the transferred heat flows through the fin.
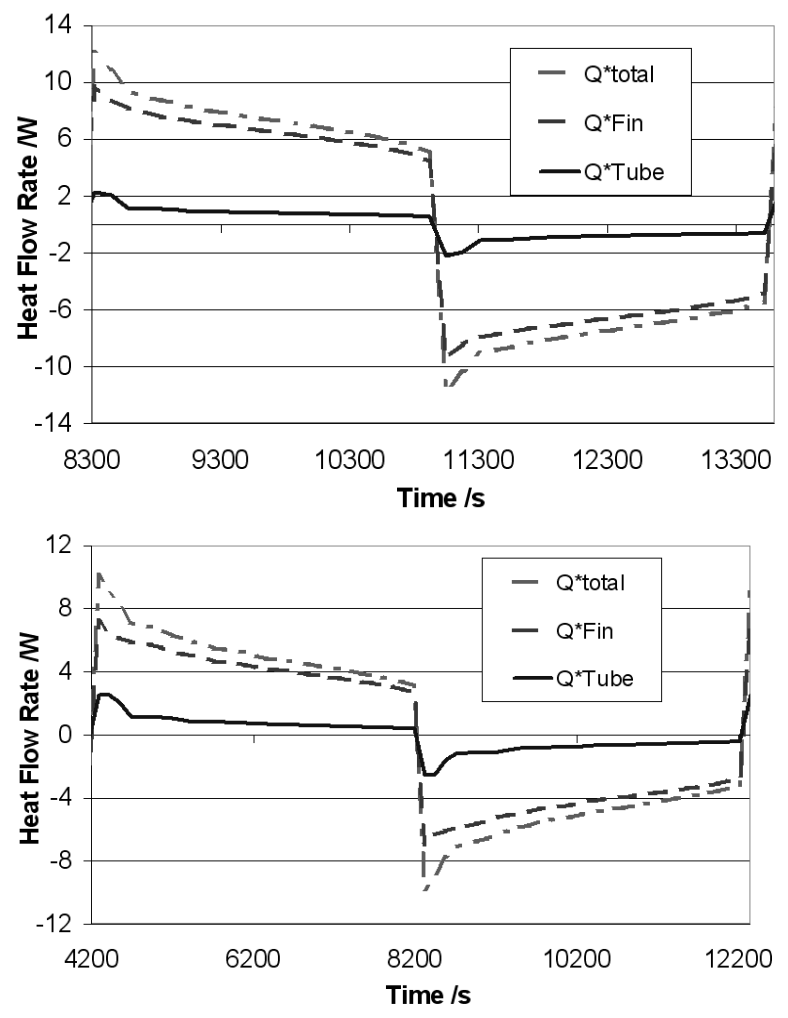

Figure 7: Heat Flow Rates in different Fin Configurations

The tube, fin and PCM mass is the same in both cases. Therefore, the amount of total enthalpy is the same. In the first case, which allows the higher transfer rate, the fins have half the thickness compared to the second case.

With the same amount of material, about $50 \%$ higher heat flow rate is achieved.

\subsection{Modeling of a whole Evaporator Storage}

In the next step, the tube-fin-PCM-model is multiplied along the tube within a discrete element of the fluid flow. Because of the almost constant temperature of a fluid when condensing or evaporating, the axial discretisation can be relatively rough. This is done in the following example of a PCM-storage evaporator unit (see Figure 8).

While discharging, the source supplies the storage module with almost saturated water. The fluid exits the storage with a mass fraction of saturated steam at the outlet. This mass fraction is separated in a steam drum. The saturated steam flows into the sink. The remaining water is recirculated through the storage by a pump.

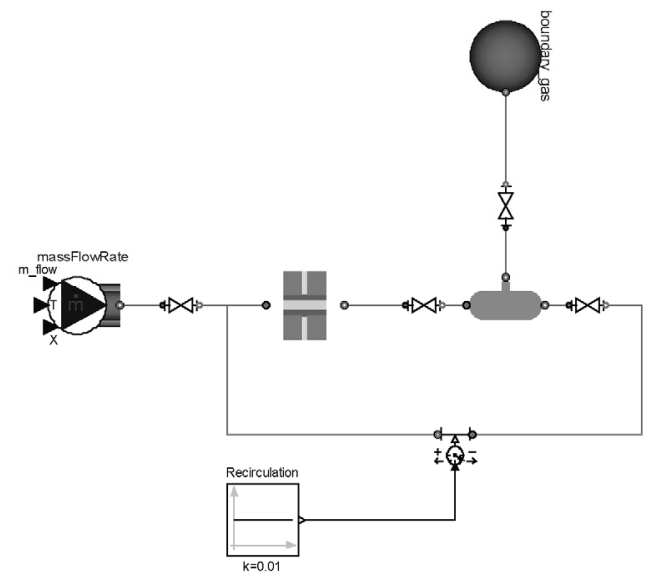

Figure 8: Example of a PCM-Evaporator Model

\section{Conclusion and Outlook}

A model to design a PCM storage equipped with finned tubes was developed. Rating and characteristic can be calculated. Further, this model is adequate to be coupled with other components, e.g. from the Modelica Fluid Library, to model whole systems.

As depicted in Figure 8, the source and sink used to simulate the sensible storages so far as shown in Figure 1 will be replaced by sensible storage models using coupled differential equations. They can be solved easily in Modelica after transformation into a system of differential algebraic equations (DAEs).

\section{References}

[1] Steinmann, W.D., Eck, M. Buffer storage for direct steam generation. Solar Energy, 2006.

[2] Buschle, J., Steinmann, W.D., Tamme, R. Analysis of steam storage systems using Modelica. 5th International Modelica Conference, 2006.

[3] Baehr, H.D., Stephan, K. Wärme- und Stoffübertragung. Springer-Verlag Berlin Heidelberg, 2006

[4] Steinmann, W.D., Buschle, J. Analysis of thermal storage systems using Modelica, 4th International Modelica Conference, 2005. 